\title{
Effects of short-term isolation on social animals' behavior: an experimental case study of Japanese macaque
}

\author{
Morita $\mathrm{T}^{1,2, *}$, Toyoda $\mathrm{A}^{3}$, Aisu $\mathrm{S}^{2}$, Kaneko $\mathrm{A}^{2}$, Suda-Hashimoto $\mathrm{N}^{2}$, \\ Adachi $\mathrm{I}^{2}$, Matsuda $\mathrm{I}^{3,4,5,6}$, Koda $\mathrm{H}^{2, \uparrow}$
}

\begin{abstract}
One of the goals in animal socioecology is to understand the functions and dynamics of group living. While observations of free-ranging animals are a crucial source of information, an experimental investigation that manipulates the size or composition, or both, of animal groups in captivity can also bring complementary contributions to the research inquiry. When paired with an automatic data collection by biologging technology, experimental studies on captive animals also allow for big data analyses based on recent machine learning techniques. As an initial exploration of this research paradigm, the present study inquired to what extent isolation of captive Japanese macaques (Macaca fuscata) changed their movement patterns. Using three-dimensional location trajectories of the macaques that were systematically collected via Bluetooth Low Energy beacons and a deep neural network, we estimated the identifiability of whether a macaque was behaving in isolation or in group. We found that the neural network identified the isolation vs. in-group conditions with more than $90 \%$ accuracy from a five-minute location trajectory, suggesting that the isolation caused notable changes from the canonical group-living behaviors. In addition, the isolation made each individual more identifiable from one another based on their location trajectories.
\end{abstract}

Keywords: biologging; convolutional neural network; individual recognition; movement ecology; primate; sociality; synchrony

\section{Introduction}

Many animals live in bonded social groups that differ in size, composition, and cohesion. To better understand proximate mechanisms behind social systems of animals as well as evolutionary factors deriving the systems, researchers have studied the costs or benefits, or boths, of group living. For example, living in a large group may reduce predation risk while competition among members for

1. Institute of Scientific and Industrial Research, Osaka University

2. Primate Research Institute, Kyoto University

3. Chubu University Academy of Emerging Sciences

4. Wildlife Research Center of Kyoto University

5. Japan Monkey Centre

6. Institute for Tropical Biology and Conservation, Universiti Malaysia Sabah

*tmorita@alum.mit.edu

$\dagger$ koda.hiroki.7a@kyoto-u.ac.jp 
food resources would become more intensive [1]. One way of addressing this research question is to observe free-ranging animals that regularly split into smaller groups (subgroups) during a day (so-called the fission-fusion social system). As an example, the correlation between the size of subgroups and different behavioral strategies taken by their members has been studied intensively [2-4]. However, collecting data from free-ranging animals is challenging due to many uncontrollable environmental factors. Specifically, there is little hope to exhaustively observe all subgroups that are theoretically possible or of interest. Also, observations of free-ranging animals are susceptible to the inevitable biases of the observers. In contrast, studies of captive animals make the size and composition of subgroups more controllable via experimental separation/isolation of group members. Thus, experiments in a captive environment can provide complementary contributions and a deeper understanding of group living. Similar experiments are common in the animal psychology field; for example, social isolation has been used to study the effects of early rearing conditions on subsequent social behaviors [5-7].

As an initial exploration of the inquiry into the effects of isolation/separation on animal behavioral patterns, this study performed a quantitative evaluation of changes in movement patterns of captive Japanese macaques (Macaca fuscata) experimentally caused by their isolation. Specifically, we trained an artificial neural network to estimate the identifiability of whether a macaque was behaving in isolation or in group based on their location trajectories. The high identifiability of isolation would indicate that the macaques exhibited some movement patterns characterizing the isolation, and using machine learning techniques, such characteristic movements can also be visualized [8]. The location records of each macaque were automatically collected in three dimensions (3D) using a recently developed biologging technology. Biolog data are systematic, fine-grained, and large-scale compared to traditional ecological observations. Therefore, they are ideal for machine learning-based and other data-scientific analyses [9-11]. Besides measuring the extent of overall behavioral changes upon isolation, we also investigated whether isolation intensifies or diminishes the macaques' individuality reflected in their movement, performing individual recognition based on the location trajectories [12]. On the one hand, if group-living restricts behavioral patterns, it is possible that isolation increases the individual recognition score by removing the bounds. For example, Japanese macaques form matrilineal groups based on social bonding among females, and these are known to exhibit greater spatial cohesion than males in general $[12,13]$. Thus, location trajectories of female macaques can look similar to one another, which would make the movement-based individual recognition difficult. However, it is also possible that isolation decreases the individual recognition score if each individual plays a specific role in the group, which reflects their individuality. In such cases, isolation would remove informative cues and thus harm individual recognition. The present study aims to adjudicate between these two possible scenarios.

\section{Materials \& Methods}

\section{Data}

We studied five adult captive Japanese macaques (two males and three females) at the Primate Research Institute, Kyoto University (KUPRI), Japan. Before the experiment, the primates were kept in an outdoor holding cage in the same group of five). The macaques were then moved to an 
experimental area, which consisted of two outdoor cages of the same size $(5 \times 4 \times 2.5 \mathrm{~m}$; see Fig. 1$)$. The two cages were adjacent to each other and partitioned by a wall with a gate. One of the cages was equipped to record the 3D location data of the macaques inside. We shut one or all the subjects in that cage by keeping the gate closed during the recording. Otherwise, if the gate was open, the subjects were allowed to freely move between the two cages. The macaques were fed primate pellets with supplemental vegetables once a day under the standard nutritional requirements for non-human primates.

The 3D location trajectories of the macaques were recorded using a real-time tracking system based on Bluetooth ${ }^{\circledR}$ Low Energy (BLE) beacons (Quuppa Intelligent Locating System ${ }^{\mathrm{TM}}$ ). Each macaque carried three BLE beacons attached to a custom-made collar. The signals from the BLE beacons were collected via six receivers. Four receivers were installed above the cage, and two on the $y=0$ side of the cage (see Fig. 1).

Each of the five macaques was isolated for six to eight hours per day $\times$ four days (see Table 1 for the dates of the recordings). The isolation started between 9:00 and 10:00 by letting the non-target macaques move to the non-recording cage and then closing the gate between the two cages. The isolation lasted until the gate was reopened between 16:00 and 17:00. We analyzed the data between 10:00 and 16:00 on each day. The isolation was performed on weekdays, while all macaques were kept together in the recording cage during weekends, which allowed us to collect group-behaving data. Four non-rainy days were selected for the analysis from weekends between February 8 and 23, (most of the isolation data were collected on weekdays in the period). Only one of the twenty weekdays was rainy and thus we excluded the rainy weekend days from the analysis. Just like the isolation data, we used the 10:00-16:00 portion of the group-behaving data for the analysis. The battery of the BLE beacons died before we collected all the data as initially scheduled. This setback caused a delay of approximately one month between the last and penultimate days of the recording (April 7 for Female 2 and March 4 for Male 1, respectively) since it took about a month to replace them and start the recording again.

\section{Analysis}

Our primary question was whether the isolation caused any changes in the macaques' 3D location trajectories compared to the in-group baseline. We addressed this question by computing the identifiability of the isolation vs. in-group condition from a location trajectory of a macaque under the condition. Specifically, we trained a convolutional neural network (CNN) that took a five-minute location trajectory of a macaque as its input and made a binary prediction of whether the location trajectory was produced under the isolation or in-group condition (see Fig. 2). The input data were in $1 \mathrm{~Hz}$, where each discrete time step represented the median of the location records collected from the three beacons in $1 \mathrm{sec}$. The accuracy of the CNN's predictions on the test portion of the data that were held out from the CNN's training process defined the identifiability of the experiment conditions (specified in Table 1, amounting to $1 / 6$ of the whole data).

To further diagnose the effect of isolation, we also assessed the identifiability of individual macaques from the location trajectories [14]. We used the same CNN architecture for this assessment, except that the network outputs the individual identity predicted as the agent that produced the input trajectory. We compared the prediction accuracy based on the isolation and in-group data. 
It is to be noted that time-series data, such as human speech and text data, are often analyzed by a recurrent neural network (RNN) whose input can have variable lengths in the time dimension (see also $[15,16]$ for a more recent model of time-series processing and $[14,17]$ for applications of RNNs to biological studies). In contrast, CNNs require their inputs to have the same length (when they return a single output instead of converting the input to another time-series). Despite this inflexibility, CNNs have an advantage over RNNs in greater interpretability of their inference. Inferences made by neural networks are, generally, difficult to interpret for human researchers because they result from compositions of many atomic numerical computations rather than a series of logical decisions. Thus, researchers need to make an extra effort to diagnose what kinds of information are used by the network, and there are visual explanation techniques exclusively available for CNNs' inferences [8,18,19]. In this study, we used Grad-CAM [8] to visualize specific segments of the location trajectories that were important for our CNNs to identify the experimental condition and individual macaque behind the data. We also weighted the Grad-CAM scores of each time step by the classification probability computed by the CNN to obtain a metric of importance for segments of different location trajectories.

\section{Results}

\section{Identifiability of Experimental Condition}

Using the neural network-based classifier, the experimental condition-isolated or in-group-was identifiable from a five-minute location trajectory of the macaques with an accuracy of $92.71 \%$ (Table 2). This result suggests that isolation robustly changed the macaques' behavioral patterns compared to the canonical group condition. Male 1 was the single subject that exhibited a notably lower accuracy $(80.26 \%)$ than the others $(93.71 \%-98.75 \%)$, though this minimum score was still higher than expected by chance $(=50 \%)$.

The identifiability evidenced robust differences between the macaques' movements under isolated and in-group conditions. However, these were unrecognizable for human researchers (see Fig. 3A, B), until Grad-CAM visualization [8] of characteristic portions of the data helped locate where the differences reside (Fig. 3C). Specifically, it revealed that macaques in isolation were characterized by their staying around the wall that separates them from the other group members ( $x \approx 5$; see also Fig. 1 for the configuration of the experimental cage).

\section{Effects of Isolation on Identifiability of Individuals}

The behavioral changes caused by the isolation also increased the identifiability of the individual subjects from their movements. Under the isolated condition, the CNN classifier recognized individual macaques in isolation with an accuracy of $87.44 \%$ (based on their five-minute location trajectories), but, when they behaved in group, the accuracy was only $62.27 \%$ (Table 3 ). This increase in individual identifiability was observed in all macaques. Grad-CAM visualization of the characteristic location trajectories of each subject is provided in the supporting information S3. 


\section{Discussion}

The present study compared location trajectories of captive Japanese macaques behaving in isolation vs. in group. The results demonstrated that the two experimental conditions were identifiable (with over $90 \%$ of accuracy) based on five-minute trajectories with the help of an artificial neural network. The result suggests that the macaques showed substantial changes in their movement patterns when isolated from other group members. Specifically, Grad-CAM visualization of characteristic movements for each condition revealed that the isolated macaques frequently visited the wall partition, where on the other side the other group members resided. Although the macaques would seemingly be well habituated for temporal isolation, we still cannot deny the possibility that the isolated individuals merely waited for the opportunity to rejoin the group. Future studies should consider such habituation effects and regard further habituation to the different experimental conditions.

The notable exception was a male (Male 1) that showed a lower accuracy of the condition classification than other individuals, though the score was still far beyond the chance level $(80.26 \% \gg$ $50 \%$ ). We suspect that this result is potentially related to the male's social status in the group. Female-philopatric/male-dispersal species like Japanese macaques tend to exhibit greater spatial cohesion among females than among males [12,13]. Our previous study of the same group also showed greater statistical dependence among the females [20]. Thus, males are expected to move more freely from social constraints than females and be less influenced by the isolation, although the dominance hierarchy among males in the group still constrained their behaviors [21,22]. A supplementary analysis of video recordings (24 hours of observation, daytime on Aug. 17-20, 2020) detected five incidences of presenting behavior by Male 2 toward Male 1 as well as one incidence of mounting behavior by Male 1 over Male 2, which indicate dominance of Male 1 over Male 2 [23]. Being the dominant individual in the group, Male 1 may be allowed to move relatively freely even under the in-group condition, whereas the movement patterns of the other subordinates were more constrained in group. In short, thanks to his dominant social status, Male 1 need not change their movement patterns depending on the experimental conditions, which resulted in the lower accuracy of the condition classification.

From the behavioral changes caused by isolation, the identifiability of individual subjects based on their movements increased. Individual recognition from movement data was more difficult in the in-group condition, in which the activity synchrony of the macaques is a factor to consider. Activity synchrony is one of the keys to enhancing group cohesion in group-living animals [24], and such synchrony has been widely reported in primates, typically in the context of feeding [25-27]. Behavioral synchrony upon feeding is expected to be more frequent in a captive environment than in wild situations. While preferred foods for free-ranging macaques (e.g., fruits and mushrooms) are seasonal and are distributed geographically patchily [28,29], captive feeding is regular. In this case, all the group members are fed approximately simultaneously, which provides a better opportunity for their behavioral synchrony. It is of note that behavioral synchrony can also occur in the form of social activities, such as grooming, but they are beyond the scope of the present analysis related to our location trajectory data. Thus, future studies are necessary to explore the loss of synchrony and other behavioral changes upon isolation or group split in other modalities, such as from video recordings. 
Notably, the accuracy scores of individual recognition (based on the in-group data) were robustly greater than our previous study [14]. Several factors are considered responsible for this improvement. First, we used one additional beacon per individual in the current study. Thereby, the total sampling frequency per individual was higher than in the previous one, which only used two beacons per individual. This increased sampling frequency enabled us to use more stable data by taking the median of every second, while the previous study used the raw samples as the inputs to the model. Likewise, the median data contained a constant number of discrete time steps for each five-minute input, which allowed us to use the CNN-based classifier. The previous study, on the other hand, used an RNN-based classifier because the input time series contained a variable number of time steps. In this study, the model inputs were also longer in duration $(5 \mathrm{~min})$ than in the previous $(2 \mathrm{~min})$; therefore the model had a better chance of finding clues to identify individuals. Note that the data analyzed here were collected more than one year after the previous, and it is possible that there has been a change in the macaques' behavioral patterns during the time.

As demonstrated here, the combination of behavioral experiments and modern technologies such as biologging systems (e.g., BLE beacons and GPS) and neural network analysis helps shed light on hitherto unrecognized latent factors/mechanisms behind behaviors of social animals. Future studies are necessary to explore a wider variety of split patterns (e.g., making subgroups of two or three members) to elucidate such complex social interactions.

\section{Acknowledgments}

This study was funded mainly by the JST Core Research for Evolutional Science and Technology 17941861 (\#JPMJCR17A4) and partially by MEXT Grant-in-aid for Scientific Research on Innovative Areas \#4903 (Evolinguistic; JP17H06380). We appreciate the animal care given by our technicians and research assistants as well as their suggestions and support for this project. In particular, we wish to express special thanks to Norihiko Maeda, Mayumi Morimoto, and Takayoshi Natsume for the animal arrangements, and Panasonic Solution Technologies Co., Ltd. for the technical support with the equipment. This study was performed under the Cooperative Research Program at KUPRI (2018-C-27, 2019-B-27, 2020-B-17).

\section{Ethics Statement}

All procedures were reviewed and approved by the Animal Welfare and Care Committee of KUPRI (Permission \# 2018-203, 2019-208, and 2020-185) and complied with the institutional guidelines [30].

\section{Author Contributions}

Project organization: IA IM HK; Animal arrangements: HK SA NSH; Apparatus building: AT IM HK; Data acquisition: AT IM HK; Animal cares: AK AT IM HK; Data management; HK AT TM; Computational modeling: TM; Manuscript writing: IA IM HK TM. 


\section{References}

1. Terborgh J, Janson CH. 1986 The Socioecology of Primate Groups. Annu. Rev. Ecol. Syst. 17, $111-136$.

2. Coles RC, Lee PC, Talebi M. 2012 Fission-Fusion Dynamics in Southern Muriquis (Brachyteles arachnoides) in Continuous Brazilian Atlantic Forest. International Journal of Primatology. 33, 93-114. (doi:10.1007/s10764-011-9555-2)

3. Lehmann J, Boesch C. 2004 To fission or to fusion: effects of community size on wild chimpanzee (Pan troglodytes verus) social organisation. Behavioral Ecology and Sociobiology. 56. (doi:10.1007/s00265-004-0781-x)

4. Symington MM. 1990 Fission-fusion social organization inAteles andPan. Int. J. Primatol. 11, $47-61$.

5. Harlow HF, Dodsworth RO, Harlow MK. 1965 Total social isolation in monkeys. Proc. Natl. Acad. Sci. U. S. A. 54, 90-97.

6. McKinney WT Jr. 1974 Primate social isolation. Psychiatric implications. Arch. Gen. Psychiatry 31, 422-426.

7. Mitchell GD, Raymond EJ, Ruppenthal GC, Harlow HF. 1966 Long-term effects of total social isolation upon behavior of rhesus monkeys. Psychol. Rep. 18, 567-580.

8. Selvaraju RR, Cogswell M, Das A, Vedantam R, Parikh D, Batra D. 2017 Grad-CAM: Visual Explanations from Deep Networks via Gradient-Based Localization. In 2017 IEEE International Conference on Computer Vision (ICCV), pp. 618-626. IEEE.

9. Dore KM et al. 2020 Review of GPS collar deployments and performance on nonhuman primates. Primates 61, 373-387.

10. Fehlmann G, King AJ. 2016 Bio-logging. Curr. Biol. 26, R830-R831.

11. Spiegel O, Leu ST, Bull CM, Sih A. 2017 What's your move? Movement as a link between personality and spatial dynamics in animal populations. Ecol. Lett. 20, 3-18.

12. Nakagawa N, Nakamichi M, Sugiura H. 2010 The Japanese Macaques. Springer Science \& Business Media.

13. Otani Y, Sawada A, Hanya G. 2014 Short-term separation from groups by male Japanese macaques: costs and benefits in feeding behavior and social interaction. Am. J. Primatol. 76, $374-384$.

14. Morita T, Toyoda A, Aisu S, Kaneko A, Suda-Hashimoto N, Matsuda I, Koda H. 2020 Animals exhibit consistent individual differences in their movement: A case study on location trajectories of Japanese macaques. Ecol. Inform. 56, 101057.

15. Vaswani A, Shazeer N, Parmar N, Uszkoreit J, Jones L, Gomez AN, Kaiser Ł, Polosukhin I. 2017 Attention is All you Need. In Advances in Neural Information Processing Systems 30, pp. 5998-6008. 
16. Tenney I, Das D, Pavlick E. 2019 BERT Rediscovers the Classical NLP Pipeline. In Proceedings of the 57th Annual Meeting of the Association for Computational Linguistics, pp. 4593-4601. Stroudsburg, PA, USA: Association for Computational Linguistics.

17. Morita T, Koda H, Okanoya K, Tachibana RO. 2020 Measuring Long Context Dependency in Birdsong Using an Artificial Neural Network with a Long-Lasting Working Memory. bioRxiv. (doi:10.1101/2020.05.09.083907)

18. Zhou B, Khosla A, Lapedriza A, Oliva A, Torralba A. 2016 Learning Deep Features for Discriminative Localization. In 2016 IEEE Conference on Computer Vision and Pattern Recognition (CVPR), pp. 2921-2929. IEEE.

19. Wang H, Wang Z, Du M, Yang F, Zhang Z, Ding S, Mardziel P, Hu X. 2020 Score-CAM: Score-Weighted Visual Explanations for Convolutional Neural Networks. In 2020 IEEE/CVF Conference on Computer Vision and Pattern Recognition Workshops (CVPRW), pp. 111-119. IEEE.

20. Morita T, Toyoda A, Aisu S, Kaneko A, Suda-Hashimoto N, Matsuda I, Koda H. 2020 Non-Parametric Analysis of Inter-Individual Relations Using an Attention-Based Neural Network. Animal Behavior and Cognition. , 1877.

21. Sprague DS, Suzuki S, Takahashi H, Sato S. 1998 Male life history in natural populations of Japanese macaques: Migration, dominance rank, and troop participation of males in two habitats. Primates 39, 351-363.

22. Suzuki S, Hill DA, Sprague DS. 1998 Intertroop transfer and dominance rank structure of nonnatal male Japanese macaques in Yakushima, Japan. Int. J. Primatol. 19, 703-722.

23. Hanby JP. 1974 Male-malemounting in Japanese monkeys (Macaca fuscata). Anim. Behav. 22, 836-849.

24. Conradt L, Roper TJ. 2000 Activity synchrony and social cohesion: a fission-fusion model. Proc. Biol. Sci. 267, 2213-2218.

25. Agetsuma N. 1995 Foraging synchrony in a group of Yakushima macaques (Macaca fuscata yakui). Folia Primatol. 64, 167-179.

26. Harrison MJS. 1985 Time budget of the green monkey,Cercopithecus sabaeus: Some optimal strategies. Int. J. Primatol. 6, 351-376.

27. Kavanagh M. 1978 The diet and feeding behavior of Cercopithecus aethiops tantalus. Folia Primatol. 30, 30-63.

28. Hanya G. 2004 Diet of a Japanese macaque troop in the coniferous forest of yakushima. Int. $J$. Primatol. 25, 55-71.

29. Sawada A, Sato H, Inoue E, Otani Y, Hanya G. 2014 Mycophagy among Japanese macaques in Yakushima: fungal species diversity and behavioral patterns. Primates 55, 249-257.

30. Primate Research Institute, Kyoto University. 2010 Guidelines for Care and Use of Nonhuman Primates. 
bioRxiv preprint doi: https://doi.org/10.1101/2021.03.28.437096; this version posted March 29, 2021. The copyright holder for this preprint (which was not certified by peer review) is the author/funder. All rights reserved. No reuse allowed without permission. 


\section{Figures}

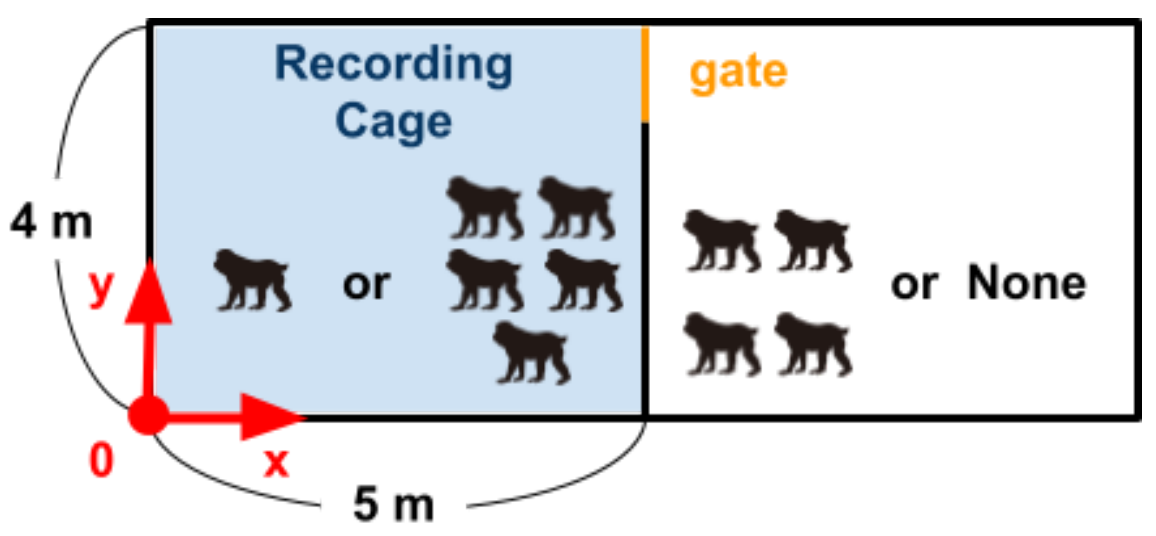

Figure 1: Recording environment. The 3D location data of one or all of the five macaques were recorded in the left cage; The other macaques were kept in the other cage on the right during the recording.

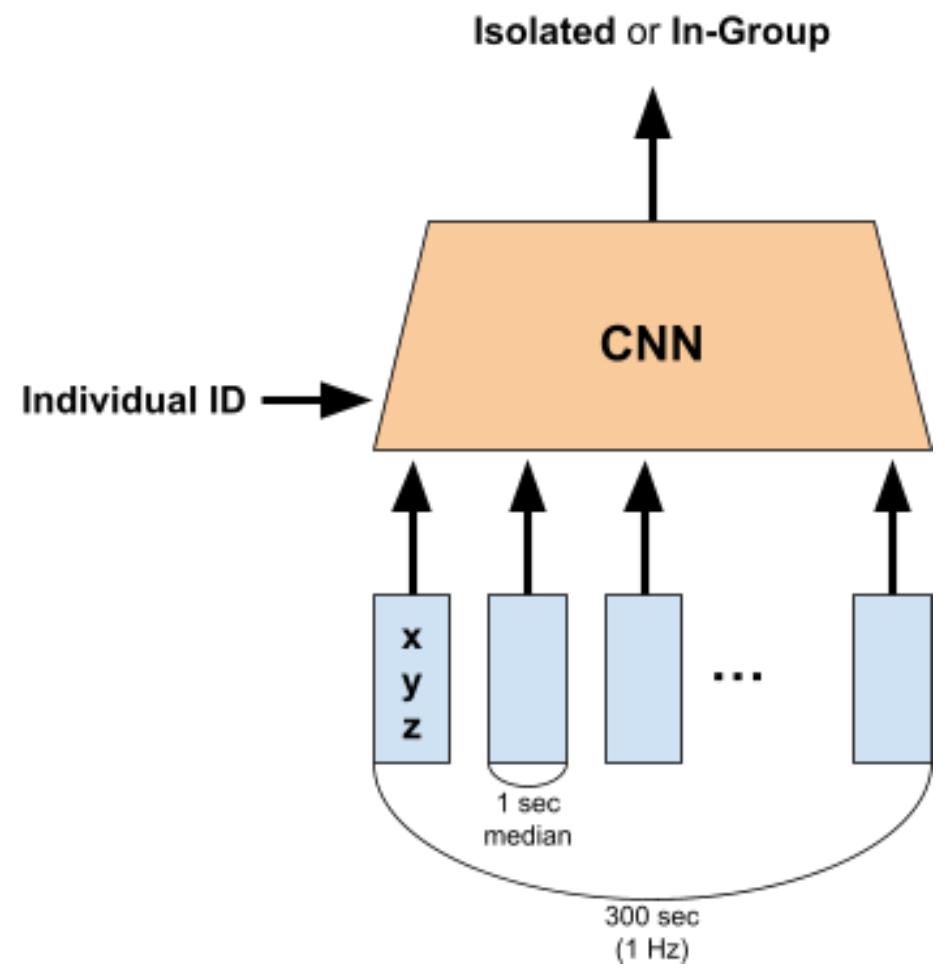

Figure 2: Overview of the model architecture. See the Supporting Information S1 for more detailed information about the CNN. The model described here predicted the experimental condition under which the input 3D location trajectory was produced. The model was also informed which individual produced the input trajectory. The individual identification was performed using the same model except that the output and background information were flipped (i.e., the individual identity was predicted from the location trajectory and the experimental condition). 

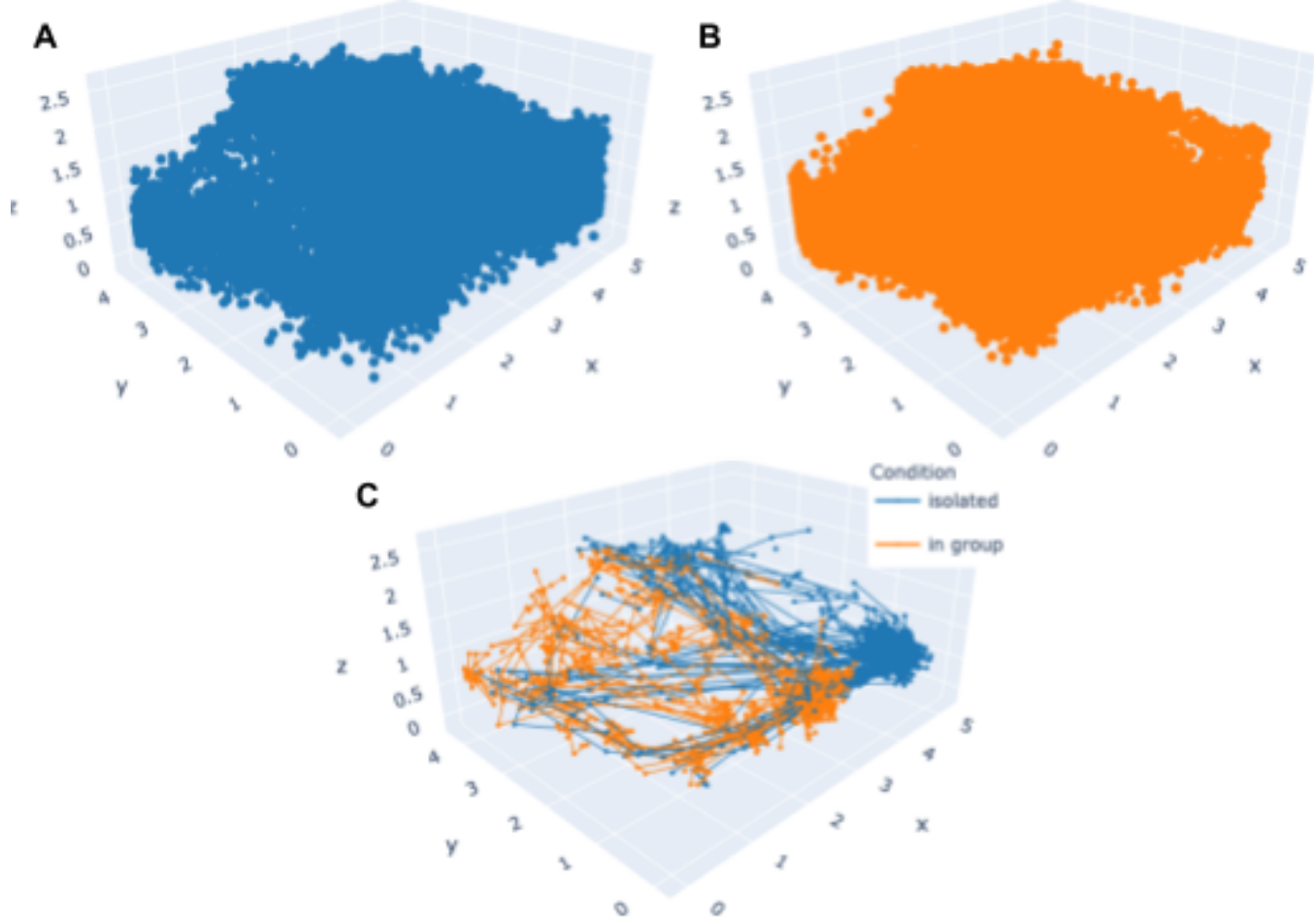

Figure 3: Scatter plot of all the locations visited by a single subject (Male 1; see the supporting information S2 for the other individuals) in (A) isolation and (B) group. (C) Grad-CAM visualization of the characteristic location trajectories of each condition [8], showing only the data yielding 0.9 or a greater value of Grad-CAM score weighted by the classification probability. 


\section{Tables}

Table 1: Dates of the recording, all in 2020. For each day, we used the 3D location data of one ("isolated" condition) or all ("in-group" condition) of the five macaques that were collected between 10:00 and 16:00. The columns tell which one-hour portion of the day was used as the test data in the analysis.

\begin{tabular}{|c|c|c|c|c|c|}
\hline \multirow{2}{*}{$\begin{array}{l}\text { Subject } \\
\text { Recorded }\end{array}$} & \multirow{2}{*}{ Condition } & \multicolumn{4}{|c|}{ Portion of data used for the test } \\
\hline & & $11: 00-12: 00$ & $12: 00-13: 00$ & $13: 00-14: 00$ & $14: 00-15: 00$ \\
\hline \multirow{2}{*}{ Female 1} & Isolated & Feb. 26 & Mar. 3 & Feb. 7 & Feb. 18 \\
\hline & In-Group & Feb. 15 & Feb. 23 & Feb. 8 & Feb. 9 \\
\hline \multirow{2}{*}{ Female 2} & Isolated & Apr. 7 & Feb. 25 & Feb. 13 & Feb. 4 \\
\hline & In-Group & Feb. 23 & Feb. 15 & Feb. 9 & Feb. 8 \\
\hline \multirow{2}{*}{ Female 3} & Isolated & Feb. 12 & Feb. 19 & Feb. 27 & Feb. 5 \\
\hline & In-Group & Feb. 9 & Feb. 15 & Feb. 23 & Feb. 8 \\
\hline \multirow{2}{*}{ Male 1} & Isolated & Feb. 6 & Feb. 14 & Feb. 21 & Mar. 4 \\
\hline & In-Group & Feb. 8 & Feb. 9 & Feb. 15 & Feb. 23 \\
\hline \multirow{2}{*}{ Male 2} & Isolated & Feb. 28 & Feb. 10 & Feb. 17 & Feb. 20 \\
\hline & In-Group & Feb. 23 & Feb. 8 & Feb. 9 & Feb. 15 \\
\hline
\end{tabular}

Table 2: Accuracy of the condition classification based on the held-out test data.

\begin{tabular}{lr}
\hline Subject & Accuracy \\
\hline Female 1 & $93.71 \%$ \\
Female 2 & $95.80 \%$ \\
Female 3 & $95.04 \%$ \\
Male 1 & $80.26 \%$ \\
Male 2 & $98.75 \%$ \\
\hline Overall & $92.71 \%$ \\
\hline
\end{tabular}


Table 3: Accuracy of the individual recognition based on the held-out test data.

\begin{tabular}{lccc}
\hline \multirow{2}{*}{ Subject } & \multicolumn{3}{c}{ Accuracy } \\
& Isolated & In-Group & Overall \\
\hline Female 1 & $87.44 \%$ & $45.89 \%$ & $66.69 \%$ \\
Female 2 & $87.17 \%$ & $71.13 \%$ & $79.15 \%$ \\
Female 3 & $92.92 \%$ & $72.94 \%$ & $82.93 \%$ \\
Male 1 & $81.84 \%$ & $43.84 \%$ & $62.84 \%$ \\
Male 2 & $87.86 \%$ & $77.57 \%$ & $82.71 \%$ \\
\hline Overall & $87.44 \%$ & $62.27 \%$ & $74.86 \%$ \\
\hline
\end{tabular}

